\title{
Seudoaneurisma de arteria peronea distal
}

\author{
Distal peroneal artery pseudoaneurysm
}

\author{
Cristiani-Winer M,* Mangupli M,* Allende-Bartolomé L,* Aguirre-Gerardo L,* Robles-Cristian O* \\ Instituto Allende de Cirugía Reconstructiva Sanatorio Allende, Córdoba, Argentina.
}

RESUMEN. Introducción: Los aneurismas y seudoaneurismas de las arterias del pie y tobillo son entidades clínicas poco frecuentes. En la mayoría de los casos ocurren a los meses o años del trauma inicial, las complicaciones en las situaciones antes mencionadas son muy infrecuentes; sin embargo, deben descartarse en los controles médicos. Presentaremos un caso clínico en el cual se produjo seudoaneurisma de la arteria peronea distal como consecuencia de una luxofractura de tobillo. Caso clínico: Paciente de 60 años de edad con trauma en tobillo derecho, su estudio radiográfico de tobillo derecho evidenció luxofractura del mismo. Se decide tratamiento quirúrgico cuatro días después de la lesión. En el postoperatorio padeció edema, ambos circulatorios de forma anormal que ameritó complemento imagenológico y se realizó arteriografía, la cual indica presencia de seudoaneurisma de $28 \times 30 \mathrm{~mm}$ aproximadamente en arteria peronea distal ameritando tratamiento específico con adecuado control de síntomas. Conclusión: Creemos que es de suma importancia la correcta valoración y examen físico de los pacientes sometidos a cirugía de tobillo en los controles postquirúrgicos sucesivos para detectar de manera precoz este tipo de complicaciones y tratarlos a tiempo.

Palabras clave: Seudoaneurisma, tobillo, complicación, diagnóstico, embolización.
ABSTRACT. Introduction: Aneurysms and pseudoaneurysms of the arteries of the foot and ankle are rare clinical entities. In most cases occur within months or years of the initial trauma, complications in the aforementioned situations are very uncommon, however, they should be ruled out at medical check-ups. We will present a clinical case in which pseudoaneurysm of the Distal Peroneal Artery occurred as a result of a fracturedislocation of the ankle. Clinical case: Patient of 60 years of age with trauma in right ankle, his radiographic study of the right ankle evidencing fracture- dislocation of the same. Surgical treatment is decided four days after the injury. In the postoperative course with edema, circulatory changes of abnormal form that merited imaging complement and arteriography was performed which indicates the presence of pseudoaneurysm of approximately $28 \times 30 \mathrm{~mm}$ in distal peroneal artery meriting specific treatment with adequate control of symptoms. Conclusion: We believe that it is of the utmost importance the correct assessment and physical examination of patients undergoing ankle surgery in successive post-surgical controls to detect these types of complications early and treat them in time.

Keywords: Pseudoaneurysm, ankle, complication, diagnosis, embolization.

\section{Introducción}

Los aneurismas y seudoaneurismas de las arterias del pie y tobillo son entidades clínicas poco frecuentes. ${ }^{1}$ En la ma-

yoría de los casos ocurren a los meses o años del trauma inicial. Se producen como consecuencia a un traumatismo como esguince de tobillo, luxación o fractura del mismo, fractura de Lisfranc, iatrogenia por artroscopía de tobillo,

* Médico adscrito al Instituto Allende de Cirugía Reconstructiva.

Correspondencia:

Mauro Cristiani Winer

Independencia Núm. 757, 1er piso, Córdoba 5000, Argentina.

E-mail: maurocw7@gmail.com

Recibido: 13-11-2019. Aceptado: 01-04-2021.

Citar como: Cristiani-Winer M, Mangupli M, Allende-Bartolomé L, Aguirre-Gerardo L, Robles-Cristian O. Seudoaneurisma de arteria peronea distal. Acta Ortop Mex. 2021; 35(3): 290-293. https://dx.doi.org/10.35366/102370

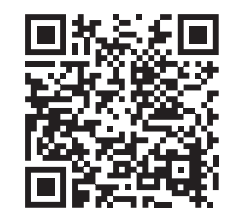



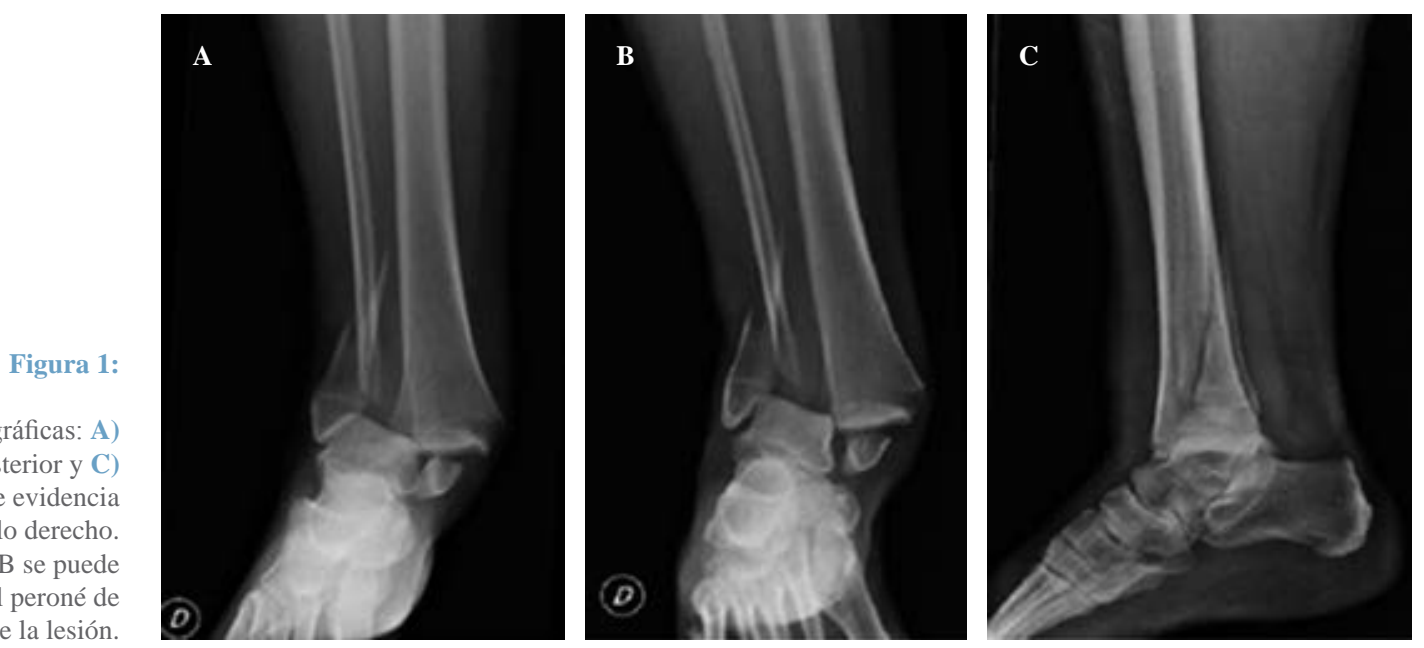

Proyecciones radiográficas: $\mathbf{A}$ mortaja, B) anteroposterior y C)

lateral, en las que se evidencia luxofractura de tobillo derecho. Tanto en A como en B se puede apreciar espícula del peroné de posible causante de la lesión.
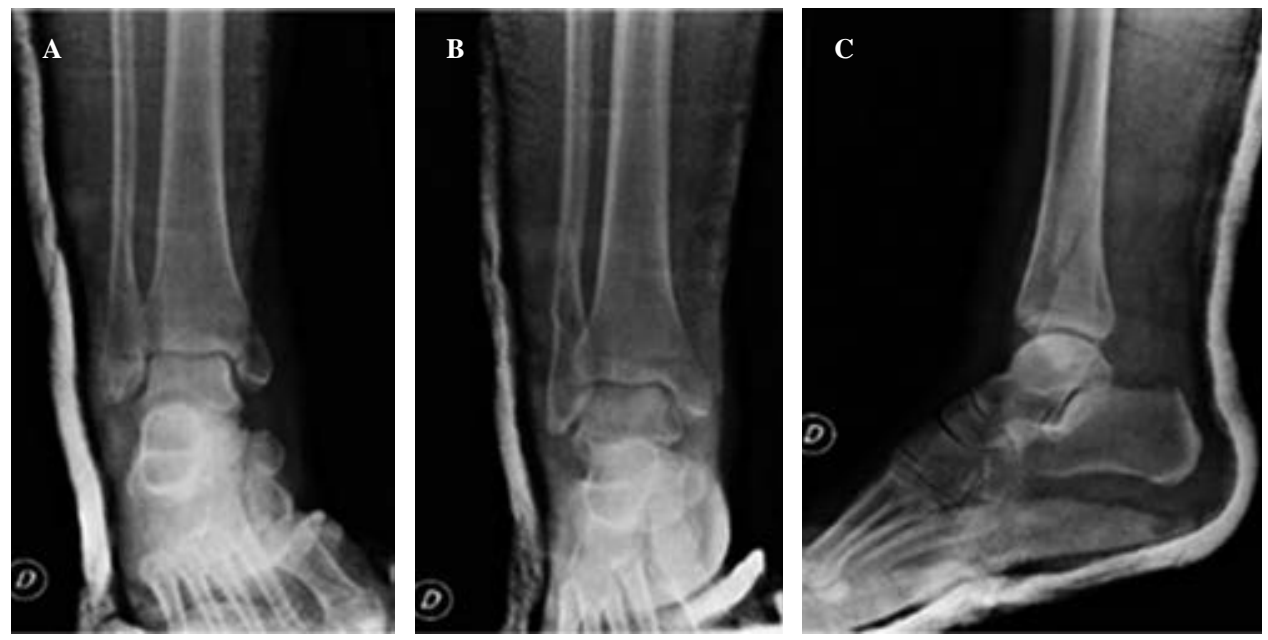

Figura 2:

Imágenes radiológicas postreducción. Proyecciones radiográficas de A) mortaja, B) anteroposterior y C) lateral.

amputación o artrodesis. ${ }^{1}$ Las complicaciones en las situaciones antes mencionadas son muy infrecuentes; sin embargo, deben descartarse en los controles médicos. En primera instancia, las alteraciones por lesión neurológica son las más comunes y luego se encuentran las complicaciones vasculares, con la formación de aneurismas o seudoaneurismas entre las posibles lesiones. ${ }^{2}$

Presentaremos un caso clínico en el cual se produjo seudoaneurisma de la arteria peronea distal como consecuencia de una luxofractura de tobillo.

\section{Caso clínico}

Paciente de 60 años de edad que consulta en guardia de emergencias por traumatismo de tobillo derecho como consecuencia de caída de propia altura. Presenta como antecedentes patológicos personales hipertensión arterial, dislipemia, hipotiroidismo y angioplastía transluminal coronaria con colocación de tres stents. Todas las patologías se encontraban bajo control mediante la administración de medicación correspondiente.
Al examen físico presenta impotencia funcional de tobillo derecho que impide la marcha. Se observa deformidad ósea e importante edema de partes blandas. Buen relleno capilar, pulsos periféricos positivos, movilidad limitada y sensibilidad con parestesias. Se decide realizar estudio radiográfico de tobillo derecho evidenciándose luxofractura del mismo (Figura 1), que correspondería a una 44-B3 dentro de la clasificación AO. A continuación, se procede a la reducción cerrada, inmovilización con valva corta de yeso en miembro inferior y posterior radiografía de control de la reducción (Figura 2). Se decide tratamiento quirúrgico programado cuatro días después, por lo que se indica tratamiento anticoagulante con enoxaparina $40 \mathrm{mg}$ y suspensión del mismo un día previo a la cirugía.

Después se realizó procedimiento quirúrgico con reducción y osteosíntesis de maléolo lateral con tornillo interfragmentario y placa de reconstrucción de huesos planos de ocho orificios; maléolo medial con tornillo rosca parcial con arandela; reducción de sindesmosis y colocación de tornillo transindesmal a través de placa. Inmovilización con férula 
de yeso. Paciente tolera la cirugía sin complicaciones con alta sanatorial al día siguiente.

El primer control postquirúrgico se realizó a los seis días de la cirugía, donde se constatan heridas con buena evolución sin signos de infección ni flogosis (Figura 3). La paciente no presentaba dolor pero sí intenso edema de miembro inferior con pulsos periféricos y sensibilidad conservada, por lo que se solicita ecografía Doppler que manifiesta signos en favor de trombosis venosa aguda focal y segmentaria. Aquel estudio se informó de manera errónea, ya que lo ubicaba en topografía de vena tibial posterior, sin signos de recanalización. Se modifica medicación anticoagulante: rivaroxabán $15 \mathrm{mg}$ cada 12 horas y se inmoviliza con bota corta de yeso.

Se cita siete días después para control ecográfico ya con ausencia de signos de trombosis venosa pero se evidencia linfedema infrapatelar severo acompañado de formación circular de $2.56 \times 2.62 \mathrm{~cm}$ de diámetro con contenido hipoecogénico y señal Doppler de flujo sistodiastólico en cara
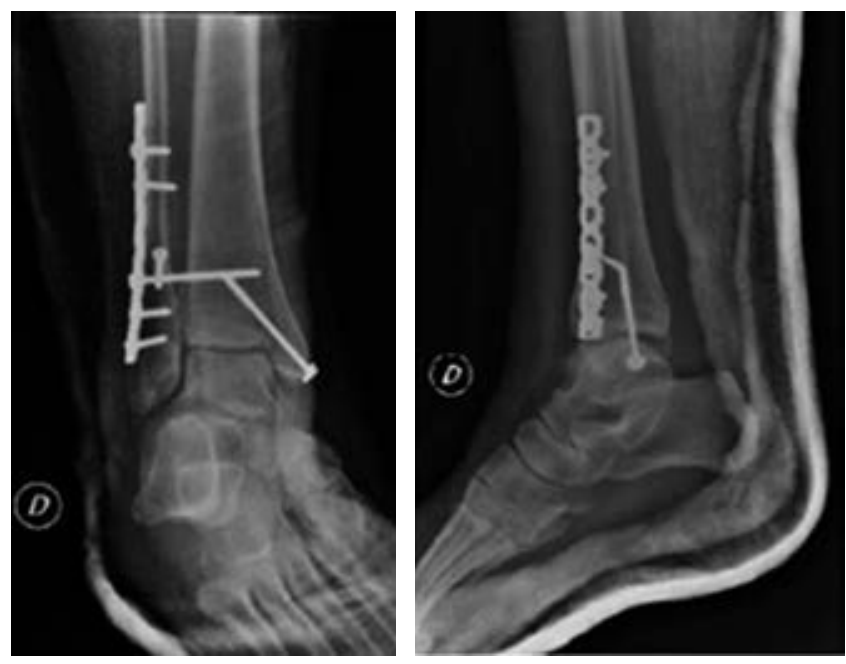

Figura 3: Par radiográfico de tobillo, proyecciones de mortaja y lateral postquirúrgicos.
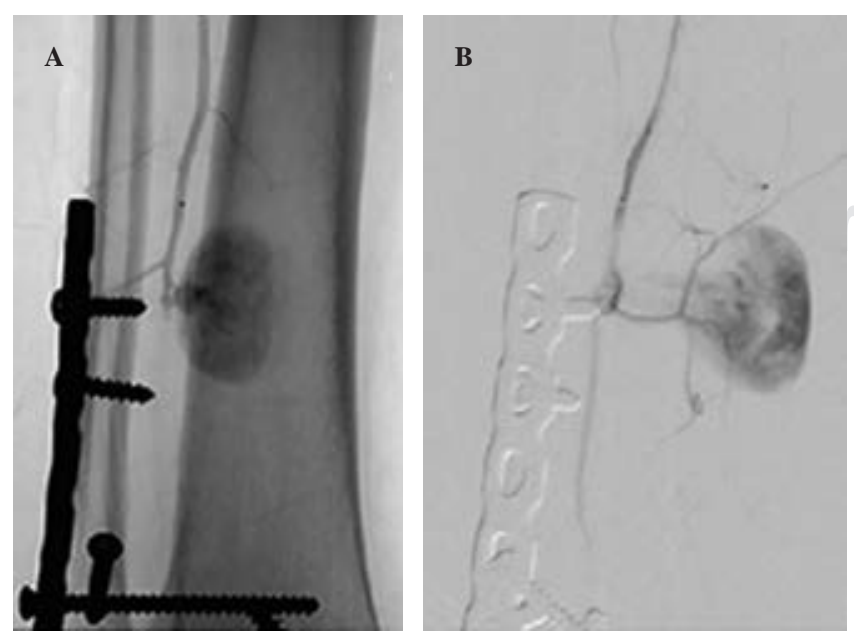

posterior de tercio distal de pierna. La mayor parte de esta formación permanecía vascularizada salvo la porción más caudal que estaba trombosada, lo cual representa signo sugestivo de seudoaneurisma parcialmente trombosado.

Se instaura rivaroxabán nuevamente a $20 \mathrm{mg} /$ día. Se indica vendaje y bota Walker y se programa arteriografía, la cual indica presencia de seudoaneurisma de $28 \times 30 \mathrm{~mm}$ aproximadamente en arteria peronea distal. Se realiza embolización de la misma con 4 coils y se controla con nueva angiografía que evidencia completa oclusión de la arteria en cuestión y la exclusión total del seudoaneurisma (Figura 4). Se punzó arteria femoral de manera anterógrada, además de retirar set introductor sin complicaciones. Se indica reposo absoluto de la pierna.

\section{Discusión}

Los seudoaneurismas de las arterias del pie y tobillo son complicaciones raras con una incidencia baja, por lo que existe gran escasez de literatura científica. ${ }^{1,3,4}$ En particular sobre la afectación de la arteria peronea distal, no hemos encontrado reportes a diferencia de otras arterias del pie como la arteria dorsalis pedis, arteria tibial anterior, entre otras. La localización de la lesión depende del mecanismo del trauma. ${ }^{1}$

Dentro de la literatura encontrada, Abdullah Alhaizaey y colaboradores propusieron una clasificación según la etiología de los aneurismas en dos grandes categorías: aneurismas verdaderos y seudoaneurismas. Los primeros ocurren en pacientes de mayor edad con ateroesclerosis como patología de base; mientras que los segundos se producen secundarios a lesión traumática, por lo cual en muchas ocasiones no se diagnostican. La localización de estos últimos dependerá del mecanismo lesional.

Por otro lado, Marcin K Kolber y su equipo establecieron una clasificación de los seudoaneurismas, también según su etiología, en postraumáticos a partir de esguinces o trauma directo, intraoperatorios como consecuencia de cirugía artroscópica o de Lisfranc y postquirúrgicos producidos por
Figura 4:

Imágenes de arteriografía. A y B) Muestran la posible relación entre el fresado que se realizó para colocar el tornillo más proximal y el nivel de seudoaneurisma. C) Imagen postratamiento en coils. 
la fijación interna que se utilizó para tratamiento de alguna fractura en la región. ${ }^{6}$

En nuestro caso clínico, consideramos que el seudoaneurisma se pudo haber producido como consecuencia al tipo de luxofractura, la cual presentaba una pequeña espícula, con la posibilidad de que en el momento de la fractura o durante la maniobra de reducción de la misma se haya dañado la indemnidad de la pared vascular. También es importante no descartar la posibilidad de que la lesión sea fruto de la perforación ósea realizada con mecha de $2.7 \mathrm{~mm}$ para la colocación del tornillo más proximal en la placa de reconstrucción, debido a la proximidad de la punta del mismo con el origen del seudoaneurisma.

Las opciones para establecer el diagnóstico incluyen Eco-Doppler, tomografía computarizada, angiografía y arteriografía. El Eco-Doppler permite confirmar cuál es la arteria que origina la masa, mientras que la tomografía y la resonancia magnética permiten definir la localización topográfica del seudoaneurisma y planificar la cirugía. La arteriografía tiene utilidad diagnóstica y terapéutica.

Las estrategias de tratamiento que deben manejarse durante la evaluación del paciente en los casos de seudoaneurismas deben incluir embolización percutánea, ligadura o excisión quirúrgica con reanastomosis. La selección del tratamiento adecuado incluye varios factores como la anatomía vascular del pie y tobillo, presencia o ausencia de ramas colaterales y asociación a fístula arteriovenosa. ${ }^{5,6}$

\section{Conclusión}

Creemos que es de suma importancia la correcta valoración y examen físico de los pacientes sometidos a cirugía de tobillo en los controles postquirúrgicos sucesivos para detectar de manera precoz este tipo de complicaciones y tratarlos a tiempo, así como tener en cuenta que el fresado del hueso con las mechas son posibles agentes lesivos de las paredes vasculares y, por ende, introducir la mecha más allá de la cortical distal puede tener repercusiones clínicas importantes.

Referencias

1. Vlachovsky R, Staffa R, Novotny T. Pseudoaneurysm of the dorsalis pedis artery: case report and literature review. J Foot Ankle Surg. 2017; 56(2): 398-400.

2. Kashir A, Kiely P, Dar W, D’Souza L. Pseudoaneurysm of the dorsalis pedis artery after ankle arthroscopy. Foot Ankle Surg. 2010; 16(3): 151-2.

3. Bozio G, Tronc F, Douek P, Bozio A, Louis D. Dorsalis pedis artery pseudoaneurysm: an uncommon cause of soft tissue mass of the dorsal foot in children. Eur J Pediatr Surg. 2009; 19(2): 113-6.

4. Williams JC, Roberts JW, Yoo BJ. Dorsalis pedis artery pseudoaneurysm after Lisfranc surgery. J Orthop Trauma. 2010; 24(11): e98-101.

5. Alhaizaey A, Hussain MA, Aljabri B, Al-Omran M. Aneurysms of the foot arteries. Vascular. 2016; 24(1): 109-12.

6. Kolber MK, Shukla PA, Kumar A, Berkowitz E. Endovascular treatment of a dorsalis pedis artery pseudoaneurysm. Vasc Endovascular Surg. 2016; 50(4): 283-5. 